\title{
Upgrading Multilevel Full Cost Allocation: Case Transport
}

\author{
Z. Bokor ${ }^{1}$, B. Horváth ${ }^{2}$ \\ ${ }^{1}$ Budapest University of Technology and Economics, Department of Transport \\ Technology and Economics \\ Müegyetem rkp. 3., 1111 Budapest, Hungary \\ Phone: +36 14631054 \\ e-mail: zbokor@kgazd.bme.hu \\ ${ }^{2}$ Széchenyi István University, Department of Transport \\ Egyetem tér 1., 9026 Győr, Hungary
}

Abstract: Multilevel full cost allocation is an effective alternative of traditional, accounting oriented costing regimes and is applied in the transport sector as well. Nevertheless, its basic algorithm may fail when the cost calculation model contains so called performance feedbacks. This is often the case in transport management practice, especially in complex operational systems. Hence effective solutions are to be found to cope with this methodological problem. This paper aims at contributing to the improvement of multilevel full cost allocation method when performance feedbacks hinder the exact calculation. The application of estimated cost ratios or specific costs is proposed and tested on a theoretical transport costing example. If some methodological requirements can be met both parameter estimations may be employed effectively in calculation tasks with performance feedbacks.

Keywords: multilevel full cost allocation, performance feedbacks, transport costing

\section{Introduction}

Activity-based costing ( $\mathrm{ABC}$ ) is a widely used costing tool. It aims at allocating indirect costs, i.e. costs that can not be assigned to products or services directly, to products or services on the basis of measured performance consumptions. Indirect costs are traced to activities first by using so called resource drivers. Then activity costs are distributed among products or services by using cost drivers, i.e. various performance indicators. By doing so a transparent and traceable cost allocation system can be set up instead of using arbitrary allocation techniques distorting cost information [7].

Multilevel full cost allocation (MFCA) is a similar method having the same advantages. Here, however, organisational units are used instead of activities for the allocation of indirect costs. These units are arranged into a multilevel hierarchy 
depicting the operational structure of the company [2]. There are transport related applications reported in the literature for both methods.

The most detailed transport oriented ABC application was carried out at a road freight transport company. Here the costs of service packages were determined and the results were compared to the outputs of traditional costing regime [1]. Road transport was in the focus of other, more complex $\mathrm{ABC}$ analyses as well [13]. $\mathrm{ABC}$ was used to calculate the costs of elementary air transport services, i.e. individual flights [14]. Furthermore, $\mathrm{ABC}$ was combined with other decision making techniques where the financial efficiency of air transport services was evaluated [11].

The cost structure of third-party logistics service providers was also analysed by ABC with special regard to transport activities [10]. The activity-based cost structure of logistics service providers focusing on road freight transport was studied by highlighting the role of fixed and variable costs [6].

MFCA has a moderate application intensity compared to the one of ABC. Sample transport cost calculation models have been developed for the cases of rail infrastructure management [2], bus transport [3], rail freight transport [4] and rail passenger transport $[5]$.

This paper focuses on multilevel full cost allocation by making also use of available research results. Besides its advantages, MFCA models used in practice still have some limitations. One of the limitations is the fact that it neglects performance feedbacks, which may reduce accuracy. Performance feedbacks mean that performance flows facilitating the operation and governing the allocation of indirect costs are not always straightforward and there may be performances that stream backwards in the system. This is the case when some organisational units have mutual performance services and their operation depends on each other's outputs. Thus the allocation of indirect costs can not be carried out exactly. This is a real problem in transport costing as the operational models establishing the calculation models, mainly in complex systems, often consist of objects interacting with each other in various ways.

Before analysing the impacts of performance feedbacks, the basic MFCA calculation model is described also with view on the latest relevant research results $[4,5]$. Note that fixed and variable costs are not separated in the applied approach as the further analysis of performance feedbacks does not require this distinction. Nevertheless, in the full scale cost calculation models the segmentation of costs into fixed and variable parts is also recommended provided the segmentation can be carried out in an exact way [6].

The basic MFCA model consists of the cost centres $(k, i=1 \ldots \mathrm{n})$ and the profit objects $(j=1 \ldots \mathrm{m})$. The former ones are the organisational units and perform for the profit objects or for other cost centres. Nevertheless, performance feedbacks, i.e. when cost centres serve each other mutually, may not be incorporated into the basic model so that the calculation can be performed in an exact way. The cost of cost centres can be calculated in the following way:

$$
C_{k}=C_{k}^{p}+\sum_{i=1}^{n} C_{i} \frac{P_{k i}}{P_{i}}
$$

where: 
$C_{k}:$ total cost of cost centre $k$;

$C_{k}^{p}$ : primary cost of cost centre $k$;

$C_{i}$ : total cost of service cost centre $i$;

$P_{k i}$ : performance consumption of cost centre $k$ at service cost centre $i$;

$P_{i}$ : total performance of service cost centre $i$.

Primary costs are assigned to cost centres directly and are obtained from the general ledger with certain transformations, if needed. Performance data are provided by technology information systems or by dedicated surveys.

Profit objects are the ultimate products or services in the examined businesstechnology system. The main goal of MFCA is to calculate the costs of profit objects. The basic formula of this calculation is the following:

$$
C_{j}=C_{j}^{d}+\sum_{i=1}^{n} C_{i} \frac{P_{j i}}{P_{i}}
$$

where:

$C_{j}$ : total cost of profit object $j$;

$C_{j}^{d}:$ direct cost of profit object $j$;

$P_{j i}$ : performance consumption of profit object $j$ at service cost centre $i$.

Direct costs are assigned to profit objects directly and are obtained from the general ledger, too. As MFCA is a full cost allocation method it is required that all indirect costs, i.e. the costs of cost centres, are fully allocated by the end of the calculation process:

$$
\sum_{k=1}^{n} \frac{P_{k i}}{P_{i}}+\sum_{j=1}^{m} \frac{P_{j i}}{P_{i}}=1
$$

As mentioned, the calculation is always based on an operational model which depicts the business and technology processes of the investigated company or organisational system. The following analysis highlights what methodological problems can be faced when the operational model and so the calculation model contain performance feedbacks.

\section{Initial problem}

The problem of performance feedbacks is illustrated using a simple transport costing example. Fig. 1 shows a simplified operation model of a transport company operating two kinds of services, i.e. regular and irregular transport services. These are the profit objects $(j=\mathrm{A}, \mathrm{B})$ in the corresponding calculation model. Four organisational units take part in the production of these services: two of them directly, i.e. vehicle pools I and II, while other two ones, i.e. operation and maintenance, indirectly. These units are regarded as cost centres $(k, i=1 \ldots 4)$. The possible performance indicators (and their dimensions) can be for example: 
- operation: disposition (number of orders);

- maintenance: service time (hours);

- vehicle pools I and II: running (vehicle kilometres).

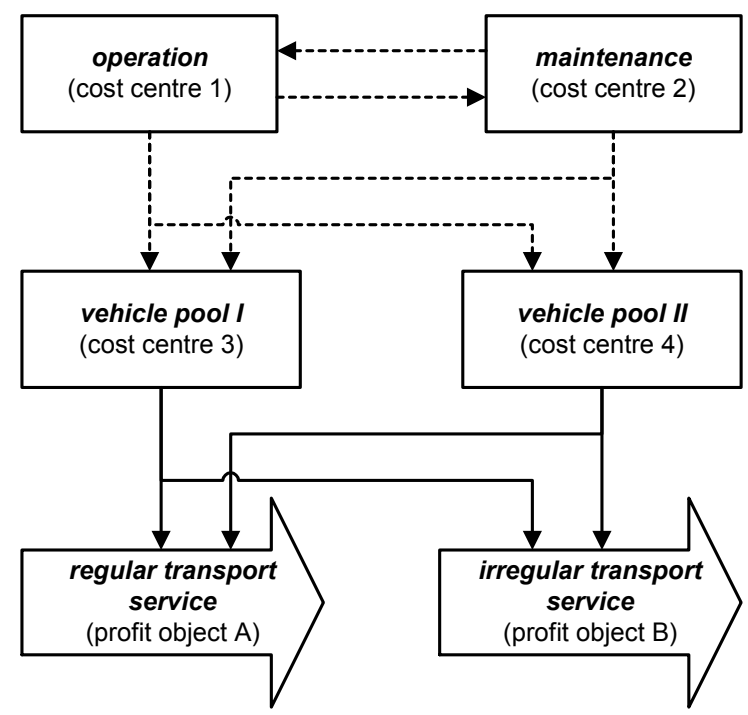

\section{Figure 1. Sample operation model}

The vehicle pools produce the transport services while the operation and the maintenance units serve the vehicle pools. The latter units serve each other as well. It means that the model contains a performance feedback in the relation of cost centre 1 and cost centre 2 .

The calculation formulas based on the extraction of (1) and (2) are the following:

$$
\begin{gathered}
C_{1}=C_{1}^{p}+C_{2} \frac{P_{12}}{P_{2}} \\
C_{2}=C_{2}^{p}+C_{1} \frac{P_{21}}{P_{1}} \\
C_{3}=C_{3}^{p}+C_{1} \frac{P_{31}}{P_{1}}+C_{2} \frac{P_{32}}{P_{2}} \\
C_{4}=C_{4}^{p}+C_{1} \frac{P_{41}}{P_{1}}+C_{2} \frac{P_{42}}{P_{2}} \\
C_{A}=C_{A}^{d}+C_{3} \frac{P_{A 3}}{P_{3}}+C_{4} \frac{P_{A 4}}{P_{4}} \\
C_{B}=C_{B}^{d}+C_{3} \frac{P_{B 3}}{P_{3}}+C_{4} \frac{P_{B 4}}{P_{4}}
\end{gathered}
$$

Let us calculate the cost of profit object $A$ :

$$
C_{A}=C_{A}^{d}+C_{3}^{P} \frac{P_{A 3}}{P_{3}}+C_{1} \frac{P_{31}}{P_{1}} \frac{P_{A 3}}{P_{3}}+C_{2} \frac{P_{32}}{P_{2}} \frac{P_{A 3}}{P_{3}}+C_{4}^{p} \frac{P_{A 4}}{P_{4}}+C_{1} \frac{P_{41}}{P_{1}} \frac{P_{A 4}}{P_{4}}+C_{2} \frac{P_{42}}{P_{2}} \frac{P_{A 4}}{P_{4}}
$$


It is obvious that the calculation cannot be executed on a regular basis because $C_{l}$ and $C_{2}$ depend on each other as can be seen in (4) and (5). Additional considerations are needed to overcome this methodological problem and to calculate the costs of profit objects.

\section{Proposed solutions}

\subsection{Estimation of cost ratios}

One of the possible solutions is to introduce the parameter of total cost ratio $\left(r_{k i}\right)$ for the case of cost centres affected by performance feedbacks:

$$
r_{k i}=\frac{C_{k}}{C_{i}}
$$

for $k i$ representing pairs of cost centres with performance feedbacks. Let us introduce $r_{12}=C_{1} / C_{2}$ in our example. Substituting by $r_{12}$ the missing cost elements can be determined from (4) and (5):

$$
\begin{gathered}
C_{1}=\frac{C_{1}^{p}}{1-\frac{P_{12}}{r_{12} P_{2}}} \\
C_{2}=C_{2}^{p}+\frac{C_{1}^{p} P_{21}}{\left(1-\frac{P_{12}}{r_{12} P_{2}}\right) P_{1}}
\end{gathered}
$$

Using (12) and (13) for $C_{1}$ and $C_{2}$ respectively, the final cost calculation of profit objects, e.g. the one of profit object $A$ in formula (10), can be executed exactly.

Another task is to estimate the value of the parameter applied:

- the simplest estimation for total cost ratio is the ratio of corresponding primary costs. It can be an acceptable solution when the total costs do not differ from primary costs significantly or are approximately proportional to total costs. Usually this is not the case;

- an alternative estimation can be the ratio of measurable natural indicators, like staff numbers, numbers of machines or appliances, etc., of corresponding cost centres. It shall be ensured that the indicator chosen is relevant for both cost centres concerned, and furthermore, that the indicator can be regarded as a real influencing factor of costs. The latter condition can be justified by correlation analyses on the basis of historical data, provided these data are available;

- combining and extending the former approaches by using the ratios of multiple factors $(l=1 \ldots q)$ which may be primary costs or selected natural indicators. Theoretically this leads to the best estimation thanks to the multiple factors considered, provided the conditions mentioned are observed and the input data are available:

$$
\hat{r}_{k i}=\prod_{l=1}^{q} \frac{f_{l_{k}}}{f_{l_{i}}}
$$

where: 
$\hat{r}_{k i}:$ estimated value of total cost ratio $k i$;

$f_{l_{k}}:$ factor $l$ at cost centre $k$;

$f_{l_{i}}$ : factor $l$ at cost centre $i$.

In our example the total costs of operation and maintenance are to be compared. The corresponding primary cost data are available but it is not sure that they reflect the total costs. Nevertheless, some natural indicators are likely to be found for the estimation. For example, the material costs of maintenance might be recorded in the served cost centres and so the main driver of maintenance costs will be labour, staff numbers or working hours. These may be appropriate indicators for estimating the cost ratio as operation is also a labour-intensive business area. Of course, the suitability of the indicator chosen depends on the operational characteristics of the company assessed. Last but not least, if more than one indicator is found appropriate or we would like to combine the ratio of primary costs and the ratio of natural indicators the complex estimation formula (14) can also be applied here.

\subsection{Estimation of specific costs}

Another possible solution for handling the methodological problem mentioned is to introduce the parameter of specific cost $\left(c_{i}\right)$ for the case of cost centres affected by performance feedbacks:

$$
c_{i}=\frac{C_{i}}{P_{i}}
$$

for $i$ representing cost centres having performance feedbacks. We can use $c_{1}$ or $c_{2}$ in our example, let us introduce $c_{2}=C_{2} / P_{2}$. Substituting by this parameter the missing cost elements can be determined from (4) and (5) again:

$$
\begin{gathered}
C_{1}=C_{1}^{p}+c_{2} P_{12} \\
C_{2}=C_{2}^{p}+C_{1}^{p} \frac{P_{21}}{P_{1}}+c_{2} \frac{P_{12} P_{21}}{P_{1}}
\end{gathered}
$$

Using (16) and (17) for $C_{1}$ and $C_{2}$ respectively, the final cost calculation of profit objects, e.g. the one of profit object $A$ in formula (10), can also be executed exactly. The question arises again: how to estimate the value of the parameter. There are two alternatives:

- using the corresponding primary specific cost values increased by estimated percentages based on relevant practical expertise. The subjectivity of this approach can be reduced by applying group decision making methods like analytic hierarchy process (AHP) or by conducting sensitivity analyses;

- using benchmark values, e.g. the average prices of similar activities or services. As benchmark values reflect the actual prices and are probably less subjective this approach seems to be a better solution, provided the benchmark data are available and the prices offered are cost-based.

In our example the specific cost of maintenance shall be determined with the dimension of (EUR / service hour). If we have experts with reliable expertise in the field we can rely on their estimations. It is, however, reasonable to obtain benchmark values 
as prices of maintenance services may easily be obtained, e.g. by calling for bids. Nevertheless, the quotations shall be reviewed carefully to ensure their applicability in the costing model.

Concerning AHP it shall be noted that this method has an extensive application potential in transport. For example, it was successfully applied in the quality analysis of public transport systems [8,9]. These research results may also be useful when AHP is utilised for supporting cost estimations.

\section{Discussion and conclusions}

The additional procedures proposed may help improve the accuracy of multilevel full cost allocation models containing performance feedbacks, with special regards to transport related applications. Comparing the two solutions it can be concluded that the application of estimated specific costs involves, in general, less risks than the one of estimated cost ratios. This can be explained by the better availability of input data, i.e. benchmark values. Nevertheless, if the estimation is company specific, then cost ratios may be more favourable as they use multiple factors describing the operational characteristics of the particular business-technology system. So it depends on the data availability and the circumstances of the estimation which approach is worth being preferred.

Although the proposed solutions have been tested on a simple example they can also systematically be applied in multilevel cost calculation models with multiple feedbacks. Of course the complexity of additional calculations is in line with the number of feedbacks.

When using the improved MFCA model the limitations of estimations shall also be considered as the estimations may contain some errors. These errors can be reduced by using further statistical or decision supporting methods like correlation analysis or AHP. Furthermore, there are some conditions which are to be observed when applying estimated cost ratios or specific costs during the calculation process. These requirements, i.e. the suitability of selected factors or benchmarks, etc., have been discussed in detail.

It shall be noted that iterative calculation procedures may also contribute to solve the problems of performance feedbacks in MFCA models. Here initial values for the missing parameters are set and the calculation programme runs until the calculated values do not change significantly. Nevertheless, the approach proposed in this paper aims at making advantages of the available information instead of iteration. However, the two different approaches, i.e. iteration and the use of estimated cost ratios or specific costs, may be effective complementary tools of cost calculation tasks that are not calculable in exact ways. This may often be the case in complex transport systems.

And at last it is worth mentioning that if the general or overall efficiency of complex transport systems is to be assessed single cost calculations may not be sufficient. Cost is namely one of the factors determining efficiency. Here more comprehensive and nonparametric methods like data envelopment analysis (DEA) can be utilised [12]. 


\section{References}

[1] Baykasoglu A, Kaplanoglu V: Application of Activity-based Costing to a Land Transportation Company: a Case Study. International Journal of Production Economics, Vol. 116, No. 2, pp. 308-324, 2008.

DOI: $10.1016 /$ j.ijpe.2008.08.049

[2] Bokor Z: Rail Infrastructure Costing Based on Multi-level Full Cost Allocation. Periodica Polytechnica. Transportation Engineering, Vol. 40, No. 1, pp. 3-9. 2012. DOI: $10.3311 /$ pp.tr.2012-1.01

[3] Bokor Z: Cost Calculation in Transport Companies. Acta Technica Jaurinensis, Vol. 5, No. 3, pp. 253-262. 2012.

[4] Bokor Z: Cost Calculation in Complex Transport Systems. LOGI - Scientific Journal on Transport and Logistics, Vol. 4, No. 1, pp. 5-22, 2013.

[5] Bokor Z: Multilevel Full Cost Allocation Model in Transport (in Hungarian). Budapest Management Review (Vezetéstudomány), Vol. 45, No. 1, pp. 55-68, 2014.

[6] Bokor Z, Markovits-Somogyi R: Applying Activity-based Costing at Logistics Service Providers. Periodica Polytechnica. Transportation Engineering, Vol. 43, No. 2, pp. 98-105, 2015.

DOI: 10.3311/PPtr.7700

[7] Cooper R, Kaplan R S: How Cost Accounting Systematically Distorts Product Costs. In Accounting and Management - Field Study Perspectives, Editors: Burns W J, Kaplan R S, Harvard Business School Press, Boston, pp. 204-228, 1987.

[8] Duleba Sz: A Hierarchical Model to Evaluate Public Transport's Supply Quality. Acta Technica Jaurinensis, Vol. 3, No. 3, pp. 377-382, 2010.

[9] Duleba Sz, Shimazaki Y, Mishina T: An Analysis on the Connections of Factors in a Public Transport System by AHP-ISM. Transport, Vol. 28, No. 4, pp. 404-412, 2013.

DOI: $10.3846 / 16484142.2013 .867282$

[10] Griful-Miquela C: Activity-based Costing Methodology for Third-party Logistics Companies. International Advances in Economic Research, Vol. 7, No. 1, pp. 133146, 2001.

DOI: $10.1007 / \mathrm{BF} 02296598$

[11] Lin W-Ch: Financial Performance and Customer Service: an Examination Using Activity-based Costing of 38 International Airlines. Journal of Air Transport Management, Vol. 19, No. 1, pp. 13-15, 2012.

DOI: $10.1016 /$ j.jairtraman.2011.12.002

[12] Markovits-Somogyi R: Data Envelopment Analysis and its Key Variants Utilized in the Transport Sector. Periodica Polytechnica ser. Transportation Engineering, Vol. 39, No. 2, pp. 63-68. 2011.

DOI: $10.3311 /$ pp.tr.2011-2.03

[13] Nurminen T, Korpunen H, Uusitalo J: Applying the Activity-based Costing to Cutto-length Timber Harvesting and Trucking. Silva Fennica, Vol. 43, No. 5, pp. 847$870,2009$.

[14] Tsai W-H, Kuo L: Operating Costs and Capacity in the Airline Industry. Journal of Air Transport Management. Vol. 10, No. 4, pp. 271-277, 2004.

DOI: $10.1016 /$ j.jairtraman.2004.03.004 